\title{
'State of the art' in aesthetic dentistry
}

Christopher Orr

Guest Editor, BDJ Aesthetic Dentistry Series

The BDJ Upfront section includes editorials, letters, news, book reviews and interviews. Please direct your correspondence to the News Editor,

Kate Quinlan at BDJNews@nature.com. Press releases or articles may be edited, and should include a colour photograph if possible.

$\mathrm{T}$ he smile is a universal means of showing welcome, friendship and acceptance. The creation and maintenance of a beautiful smile has always been a part of dental practice. In the past, this has often presented a considerable clinical challenge, as older techniques and materials were 'technique sensitive', in that it took considerable time and outstanding clinical skill to achieve a good result.

Aesthetic dentistry has transformed itself from a niche area to an established part of mainstream practice over the last 20 years, through a convergence of favourable factors.

People are keeping their natural teeth for longer. Dentate members of older generations considered themselves fortunate by comparison to their friends who lost all of their teeth early in their lives. Advances in dental care mean that today's adults expect not only to keep most of their teeth for most of their lives, but that they should look good as well. Great improvements in dental public health have meant that in many parts of the UK, children arrive at the age of 18 or 21 - where their grandparents or great-grandparents may have been considering a clearance - with very little experience of dental disease.

We also live in an age of information. Our patients have become more and more educated about all aspects of their lives, including dentistry. Greater patient awareness has led to an increase in demand for elective treatment which has had the effect of making us more aware of, and critical about, the treatments that we can devise, offer and provide. In turn this has led to higher levels of skill from the profession. As healthcare professionals, we have always striven to provide aesthetically beautiful results for our patients in as minimally-invasive a manner as possible. The emphasis at aesthetic dentistry conferences today is 'look how little I prepped it' not the 'look how well I prepped it' of 20 years ago. In the past such considerations, while they may, or may not, have been explained in detail to the patient probably had less impact than in today's Internet-empowered world. Not only are our patients better informed they are also more likely to question the removal of hard dental tissue, being more able to understand at least the irreversibility of this procedure even if not the full lifetime consequences of it.

The move towards minimal intervention has been made possible by great improvements in restorative materials, which make it possible for a motivated practitioner to achieve a good result in a realistic amount of clinical time. Also, there has been considerable blurring of the lines between specialist and general practice, utilising a wider range of techniques to facilitate minimal intervention. The rise elective procedures and as practitioners we overlook this essential element at our cost.

New techniques may have made a difference but the age-old imperative of good communication remains as crucial as ever. As the range of available treatment modalities increases, it becomes impossible for one practitioner to provide all parts of the treatment plan. Thus the need for good communication applies equally to communication between members of the interdisciplinary dental team, who work together to provide an outcome which is better than that which would have been achieved by any individual working alone.

Thus the 'state of the art' of aesthetic dentistry today is very different from twenty years ago. The series of articles that begins in this issue of the $B D J$ will examine all aspects of

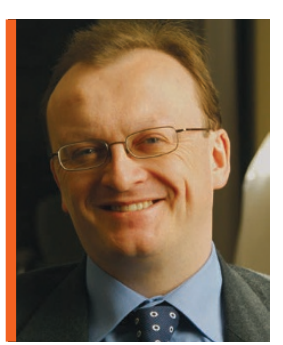

\section{'The emphasis today is 'look how little I prepped it' not 'look how well I prepped it'...'}

of GDP-provided orthodontics, whilst not universally welcomed, avoids the need for extensive tooth preparations to achieve an improved smile for the patient, and allows postorthodontic restorative care to be provided in as minimally invasive a manner as possible.

Regardless of the treatment modality, thorough diagnosis has become much more important to recognise clinical problems and areas of risk so that the treatment plan will manage the problems and control the risks in the best ways possible. Management of the patient's expectations regarding treatment times, outcomes and longevity is also critical to success.

Indeed, particularly in dento-legal terms, this can be crucial to the outcome of these current aesthetic dental practice, ranging from diagnosis and treatment planning, though to direct and indirect restorations, occlusal management, fixed/removable prosthodontics, orthodontics and patient psychology.

Acting as Guest Editor for a series like this is a great privilege but also a daunting task. Originally conceived as a $B D J$ themed issue, the preference to run the articles as a series became apparent from the wonderful response I received from my friends and colleagues who were kind enough to agree to write about the 'state of the art' in their respective areas. I thank them all for their excellent contributions, and hope that you, the reader, enjoy reading this series as much as I have in putting it together. DOI: 10.1038/sj.bdj.2016.748 